(1)

CrossMark

\title{
Associations between dietary scores with asthma symptoms and asthma control in adults
}

\author{
Roland M. Andrianasolo ${ }^{1}$, Emmanuelle Kesse-Guyot ${ }^{1}$, Moufidath Adjibade ${ }^{1}$, \\ Serge Hercberg ${ }^{1,2}$, Pilar Galan ${ }^{1}$ and Raphaëlle Varraso ${ }^{3,4}$ \\ Affiliations: 'Université Paris 13, Equipe de Recherche en Epidémiologie Nutritionnelle (EREN), Centre \\ d'Epidémiologie et Statistiques Sorbonne Paris Cité, Inserm (U1153), Inra (U1125), Bobigny, France. \\ ${ }^{2}$ Département de Santé Publique, Hôpital Avicenne, Bobigny, France. ${ }^{3}$ Inserm, U1168, VIMA: Aging and \\ chronic diseases. Epidemiological and public health approaches, Villejuif, France. ${ }^{4}$ Uniiversité Versailles \\ St-Quentin-en-Yvelines, UMR-S 1168, Montigny le Bretonneux, France.
}

Correspondence: R.M. Andrianasolo, Equipe de Recherche en Epidémiologie Nutritionnelle (EREN), 74 rue Marcel Cachin, 93017 Bobigny, France. E-mail: r.andrianasoloderen.smbh.univ-paris13.fr

@ERSpublications

Healthy diet behaviours are associated with less asthma symptoms and greater asthma control http://ow.ly/JPns30jWIYp

Cite this article as: Andrianasolo RM, Kesse-Guyot E, Adjibade M, et al. Associations between dietary scores with asthma symptoms and asthma control in adults. Eur Respir J 2018; 52: 1702572 [https://doi. org/10.1183/13993003.02572-2017].

ABSTRACT Associations between asthma and the nutritional quality of diet remain poorly understood. We investigated the associations between overall quality of diet evaluated by three dietary scores (the Alternate Healthy Eating Index 2010 (AHEI-2010), the literature-based adherence score to Mediterranean diet (MEDI-LITE) and the modified Programme National Nutrition Santé Guideline Score (mPNNS-GS)) and the asthma symptom score and asthma control.

In 2017, 34766 participants from the NutriNet-Santé cohort answered a detailed respiratory questionnaire. Asthma was defined by the asthma symptom score (as the sum of five questions) and asthma control by the asthma control test (ACT).

Roughly $25 \%$ of participants reported at least one asthma symptom and for the three dietary scores we observed a statistically significant negative association between a healthier diet and the asthma symptom score (for AHEI-2010, the adjusted OR was 0.79 (95\% CI 0.75-0.84) for women and 0.67 (95\% CI 0.60 $0.75)$ for men). Among participants with asthma $(n=2609)$ we also observed a negative association between a healthier diet and poorly controlled asthma. This was significant in men (OR 0.39 (95\% CI 0.18-0.84) for the AHEI-2010) and borderline significant in women (OR 0.73 (95\% CI 0.53-1.01) for the AHEI-2010; $\mathrm{p}=0.06$ for trend).

Healthier diet behaviours were associated with fewer asthma symptoms and greater asthma control. 


\section{Introduction}

Although asthma is one of the most common chronic diseases in the world, affecting about 358 million people [1], it remains poorly understood and targeted primary and secondary preventions are lacking. Over the last decades, the prevalence of asthma has markedly increased as a potential consequence of environmental and lifestyle changes [2], at the forefront of which is the modification of overall diet quality [3] characterised by an increased consumption of processed and refined foods, and a decreased consumption of fresh vegetables and fruits. In this context, most studies on diet-asthma association are based on specific foods or nutrients [3-5]. However, limitations have been noted about this method because foods are consumed as complex combinations, which include several nutrients and bioactive substances that interact with each other and influence bioavailability and absorption, rather than single specific foods or nutrients [6]. Diet scores based on existing nutritional knowledge have thus been proposed to assess overall diet quality, providing a quantitative measure taking into account synergic effects among nutrient and food intakes [3]. Up to now, very few epidemiological studies have been conducted on the association between overall dietary scores and asthma phenotypes in adults [7-12] and, taken together, the level of evidence is still poor. Discrepant findings are probably due to the complexity of assessment of both outcomes and exposures and, consequently, of their associations.

Regarding outcomes, only two studies investigated the role of overall diet quality as a primary prevention for asthma; however, they used different asthma definitions and reported discrepant findings [9, 12]. The asthma symptom score, which is recommended for the study of risk factors in asthma [13, 14], is particularly relevant in epidemiological studies investigating the diet-asthma association, as it can be used in participants both with and without asthma allowing integration of different asthma phenotypes and different levels of asthma prevention. As a basis for secondary prevention studies, better understanding of the reasons why asthma is getting worse in a substantial proportion of patients would be very informative. According to the latest Global Initiative for Asthma (GINA) report, a healthy diet high in fruits and vegetables is encouraged for people with asthma due to its general health benefits [15]; however, up to now, very few studies have been conducted in adults regarding the role of diet quality in asthma control and they reported discrepant results $[7,8,11,12]$.

Regarding exposure, several dietary scores have been proposed, based on prevailing hypotheses and guidance about the role of nutrients in disease prevention, in order to estimate the effects of overall diet [6]. International diet scores have been developed, including the Alternate Healthy Eating Index 2010 (AHEI-2010) and the literature-based adherence score to Mediterranean Diet (MEDI-LITE) [16]. The AHEI-2010, a measure of diet quality, has been widely used and is consistently associated with a lower risk of mortality and major chronic diseases, including chronic obstructive pulmonary disease (COPD) and asthma $[9,12,17,18]$. The MEDI-LITE has recently been proposed as a valid ambulatory instrument to assess adherence to the Mediterranean diet, which has been associated with better health [19]. Regarding national dietary scores, the modified Programme National Nutrition Santé Guideline Score (mPNNS-GS) [20] has been developed to evaluate adherence, at individual level, to the French Nutrition and Health Programme (PNNS) [21] dietary guidelines and has been associated in cohort studies with a lower risk of chronic disease and mortality [22].

In the current study, in a large French cohort of adults, we aimed to investigate 1) the association between asthma symptom score and nutritional quality of diet (as evaluated by three dietary scores, the AHEI-2010, the MEDI-LITE and the mPNNS-GS) and 2) the extent to which these dietary scores are also associated with asthma control.

\section{Methods}

\section{Study population}

Participants were volunteers, aged 18 years or older, from the NutriNet-Santé cohort, a large web-based prospective observational cohort the main objective of which is to investigate the relationship between nutrition and health and between determinants of dietary behaviour and nutritional status. The NutriNet-Santé study has been described in detail elsewhere [23]. All participants provided electronic informed consent and all procedures were approved by the Institutional Review Board of the French Institute for Health and Medical Research (IRB Inserm Paris, France; no. 0000388FWA00005831) and the French Institutional Ethics Committee (CNIL no. 908450 and no. 909216).

\section{Diet scores assessment}

At baseline and twice a year thereafter all participants were invited to complete three self-administered, validated [24], web-based 24-h dietary records randomly distributed within a 2 -week period (2 days in the week and 1 day at the weekend), recording all foods and beverages consumed throughout the day. For each participant, daily mean food consumption refers to the average consumption, as reported on all available 24-h dietary records completed during the first 2 years of follow-up, weighted for the type of day (week or 
weekend). Nutrient intakes were estimated using the NutriNet-Santé food composition table, which includes more than 3000 different items [25]. In the present study, only participants with at least three 24-h records from baseline until 2 years of follow-up were included. Dietary scores were computed and, for each score, points were assigned for each of the components using predefined cut-off points (portion sizes or recommended nutrient intakes). Among participants with reliable dietary data (figure 1), we computed three dietary scores reflecting adherence to healthy eating: the AHEI-2010 [26], the MEDI-LITE [16] and the mPNNS-GS [20] (table 1). For these three scores, a higher result represented a healthier diet and a lower result a less healthy diet. More details regarding the scoring methods are provided in the supplementary material. Correlations between AHEI-2010 and MEDI-LITE were 0.48 in women and 0.57 in men, while between AHEI-2010 and mPNNS-GS they were 0.54 in women and 0.55 in men. Finally, between MEDI-LITE and mPNNS-GS the correlations were 0.33 in women and 0.42 in men.

\section{The respiratory survey in the NutriNet-Santé cohort}

In order to improve the respiratory characterisation in the cohort, a detailed questionnaire on respiratory health based on international standardised recommendations [27] was proposed in April 2016 to all the active participants $(n=121568)$. As of June 2017, the survey had been filled-in by 40152 adults. Respondents were then compared to non-respondents (see supplementary table S1).

Ever asthma was defined by at least one positive answer to the question "Have you ever had asthma?" in main questionnaires or by a positive answer to "Have you ever had an asthma attack?" or "Have you ever had an attack of shortness of breath at rest with wheezing?” in the respiratory survey.

We used the asthma symptom score $[13,14]$ which has been proposed previously as a continuous measure of asthma in epidemiological studies. It is a validated score, ranging from 0 to 5 , based on the number of respiratory symptoms during the past 12 months.

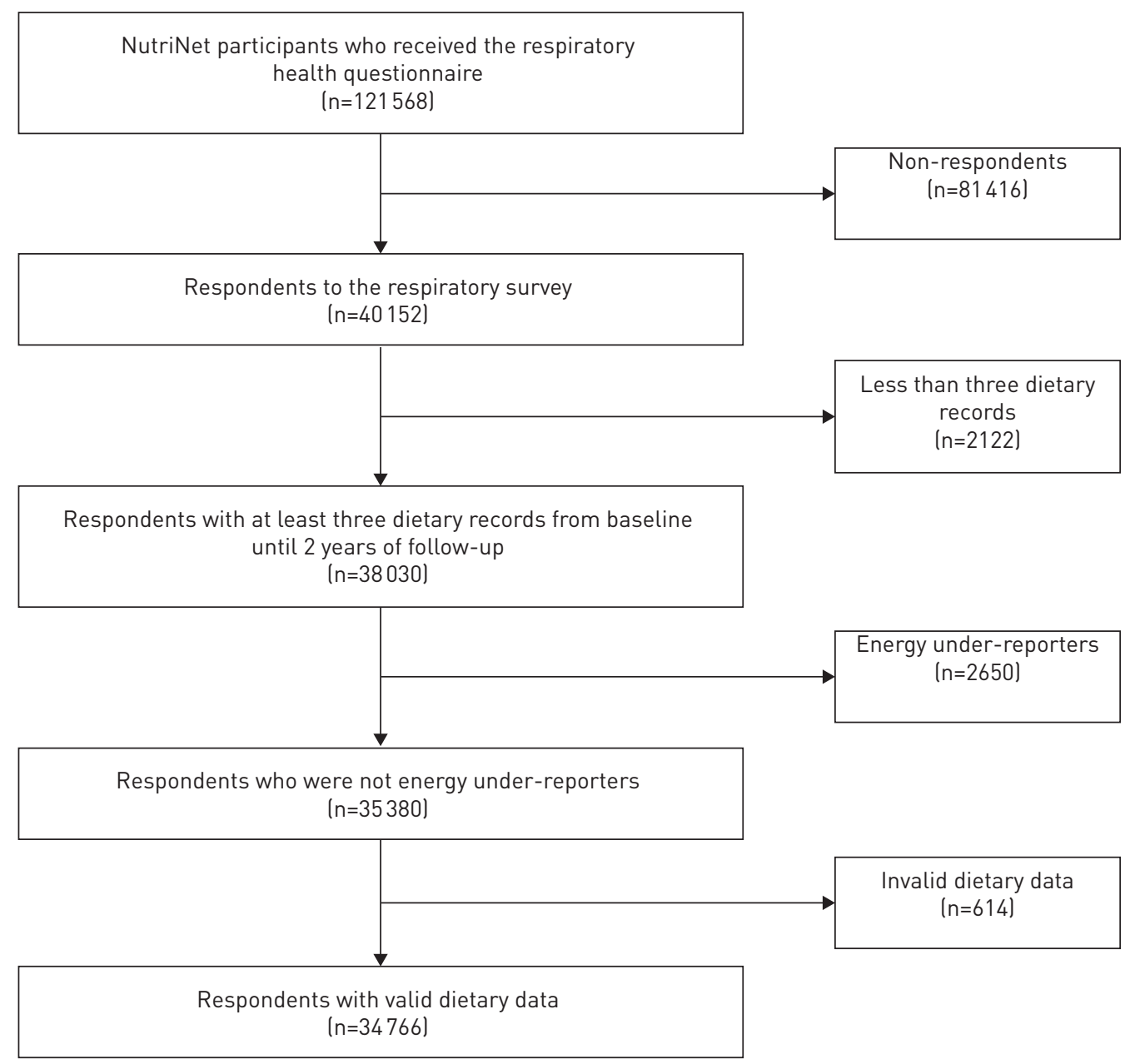

FIGURE 1 Flowchart for inclusion of participants 
TABLE 1 Description of dietary scores (the Alternate Healthy Eating Index 2010 (AHEI-2010), the literature-based adherence score to Mediterranean diet (MEDI-LITE) and the modified Programme National Nutrition Santé Guideline Score (mPNNS-GS))

Intake level

Dietary Score

AHEI-2010 MEDI-LITE

mPNNS-GS

High intakes recommended

Fruits $(0-10)$

Vegetables $(0-10)$

Nuts and legumes $(0-10)$

Whole grains $(0-10)$

Long-chain $(n-3)$ fats $(0-10)$

Polyunsaturated fatty acids $(0-10)$

Adequate intakes

Moderate intakes

Alcohol (0-10)

Low intakes recommended

Red/processed meat (0-10)

Sodium $(0-10)$

Sugar-sweetened beverages (0-10)

Total score range ${ }^{+}$

$0-100$
Fruits (0-2)

Vegetables $(0-10)$

Legumes (0-2)

Cereals (0-2)

Fish (0-2)

Olive oil (0-2)
Fruits and vegetables (0-2)

Bread, cereals, potatoes and legumes (0-1) Whole grain food $(0-1)$ Seafood (0-1)

Vegetables added fat $(0-1)$

Milk and dairy products $(0-1)$

Meat and poultry, seafood and eggs (0-2)

Nonalcoholic beverages (water and soda) (0-1)

\author{
Alcohol (0-1) \\ Sweetened foods ( -0.5 to 1$)$ \\ Added fat (0-1) \\ Salt $(-0.5$ to 1$)$
}

Values in parentheses indicate the range of points attributed to the category. " : without the trans fat component; " : without physical activity;

${ }^{+}$: a higher score corresponded to better diet quality for all of the three dietary scores.

To evaluate asthma control, we used the asthma control test (ACT), a validated self-administered questionnaire [28] based on five questions on activity limitations, frequency of symptoms, and frequency of use of quick-relief medication in the past 4 weeks. Each item is scored from 1 to 5 and the total ACT score ranges from 5 to 25 . Participants who responded positively to "Have you ever had allergic rhinitis?" or "Have you ever had hay fever?" were classified as "allergic rhinitis".

\section{Sociodemographic data}

At baseline, participants answered validated, self-administered questionnaires [29] to collect data on sociodemographic characteristics, lifestyle, physical activity and anthropometric measurements. Educational level was classified by four categories $(<13,14,15-16$ and $\geqslant 17$ years duration) and smoking status by three groups (never smokers, former smokers and current smokers). Among ever smokers, pack-years were calculated to estimate the amount of tobacco smoke. Body mass index (BMI) was calculated as weight $(\mathrm{kg}) /$ height $^{2}\left(\mathrm{~m}^{2}\right)$ and categorised according to the World Health Organization (WHO) classification [30]. Leisure-time physical activity was estimated using the validated international physical activity questionnaire (IPAQ) [31] short form and classified in three intensity bands (vigorous, moderate and low).

\section{Statistical analysis}

To account for gender differences [32] in diet-asthma association, analyses were conducted separately among women and men, and each dietary score was classified based on sex-specific tertiles. Associations between tertiles of dietary scores and the asthma symptom score were evaluated by a negative binomial regression, as has been done previously [33], with asthma symptom score being considered as a continuous variable. Main models were adjusted for age, smoking, pack-years, educational level, leisure-time physical activity, total daily energy, allergic rhinitis and family history of asthma. We also 
performed a sensitivity analysis among participants who never reported asthma from their inclusion in the NutriNet-Santé cohort until their response to the respiratory survey. In addition, since diet quality is often associated with smoking habit, we conducted a sensitivity analysis stratified on smoking status (see supplementary table S5) and formally tested the interaction between tertiles of dietary scores and smoking. As diet affects BMI and obesity is a likely risk factor for asthma, BMI might be a potential mediator in the diet-asthma association. Therefore, we formally tested the interaction between tertiles of dietary scores with BMI category $\left(<25,25-29.9, \geqslant 30 \mathrm{~kg} \cdot \mathrm{m}^{-2}\right)$ and performed a stratified analysis on BMI (see supplementary table S6).

Asthma control was at first classified into three categories (well controlled (ACT $\geqslant 20$ ), not well controlled (ACT 16-19) and poorly controlled (ACT $\leqslant 15)$ ). However, we faced a sample size issue amongst poorly controlled subjects (see table 2 and supplementary tables S2 and S3) and thus combined ACT 16-19 with ACT $\leqslant 15$ and considered asthma control under two categories (ACT $>19$ (well controlled) and ACT $\leqslant 19$ (not controlled)) [28]. Associations between tertiles of dietary scores and asthma control were evaluated by a multinomial logistic regression. Models were adjusted for age, smoking, pack-years, educational level, leisure-time physical activity and total daily energy.

Finally, to evaluate how robust our diet-asthma association was to potential unmeasured confounding, we performed a bias analysis based on the E-value, as recently published [34]. The E-value is a measure that represents the minimum strength of association that an unmeasured confounder would need to have with both the exposure and the outcome to fully explain the association. When the outcome is relatively rare $(<15 \%)$ with an odds ratio $(\mathrm{OR})<1$, the E-value is given by: E-value=1/OR+sqrt $\{1 / \mathrm{OR} \times(1 / \mathrm{OR}-1)\}$.

Missing data for covariates were handled by multiple imputations $(\mathrm{n}=10)$ according to a Markov chain Monte Carlo approach [35]. All tests were two-sided and p-values $<0.05$ were considered statistically significant. Analyses were conducted using SAS version 9.4 (SAS Institute, Cary, NC, USA).

\section{Results}

\section{Participant characteristics}

Participant characteristics are shown in table 2 for women and men according to tertiles of AHEI-2010. Both among women and men, participants in the highest tertile of the AHEI-2010 diet were significantly older when compared to participants with the least healthy diet (tertile 1). Women and men with the healthiest diet were less likely to be current smokers, had a higher educational level, were more physically active and were less likely to be obese when compared to women and men with the least healthy diet. They also reported less allergic rhinitis and less ever asthma. Similar characteristics were observed according to tertiles of MEDI-LITE (see supplementary table S2) and of mPNNS-GS (see supplementary table S3).

\section{Associations between dietary scores and asthma symptom score}

Among women, $28 \%$ of subjects reported at least one asthma symptom in the last 12 months (the corresponding figure in men was 25\%). Among both women and men, after adjustment for age, a healthier diet measured by AHEI-2010 was negatively and significantly associated with the asthma symptom score (table 3). After adjustments for potential confounders, the association remained statistically significant (figure 2). When healthy diet was assessed by the MEDI-LITE diet score, similar associations were reported both among women and men (figure 2). Finally, when healthy diet was assessed by mPNNS-GS, a negative and significant association between greater adherence to the mPNNS-GS and lower asthma symptoms was reported; however, the strength of the association decreased among women (figure 2).

For the bias analysis, E-values were 1.92, 1.92 and 1.67 among women for AHEI-2010, MEDI-LITE and mPNNS-GS, respectively (the figures in men were 2.34, 2.12 and 2.08, respectively). When analyses were restricted to participants without ever asthma ( $\mathrm{n}=23752$ (women) and $\mathrm{n}=7911$ (men)), similar associations were reported between the asthma symptom score and the three dietary scores (see supplementary table S4). After stratification based on smoking habits, associations remained significant and of similar magnitude within each stratum (see supplementary table S5). However, the interaction between diet quality and smoking was not statistically significant ( $p=0.39,0.65$ and 0.80 for AHEI-2010, MEDI-LITE and mPNNS-GS, respectively). Lastly, after stratification based on BMI, associations remained below one within each stratum (see supplementary table S6) and the interaction between diet quality and BMI was not statistically significant $(\mathrm{p}=0.17,0.23$, and 0.12 for AHEI-2010, MEDI-LITE and mPNNS-GS, respectively).

\section{Associations between dietary scores and asthma control}

Out of the 40152 women and men who responded to the respiratory survey, 3116 reported ever asthma and 2609 completed the ACT questionnaire. Asthma was not controlled (ACT $\leqslant 19$ ) in $15 \%$ of women and $11 \%$ of men. Among both women and men, those with uncontrolled asthma were significantly younger, 
TABLE 2 Characteristics of the participants, before imputation, according to the tertiles of the Alternate Healthy Eating Index 2010 (AHEI-2010) diet score among women ( $n=26$ 197) and men ( $n=8569)$ from the NutriNet-Santé study

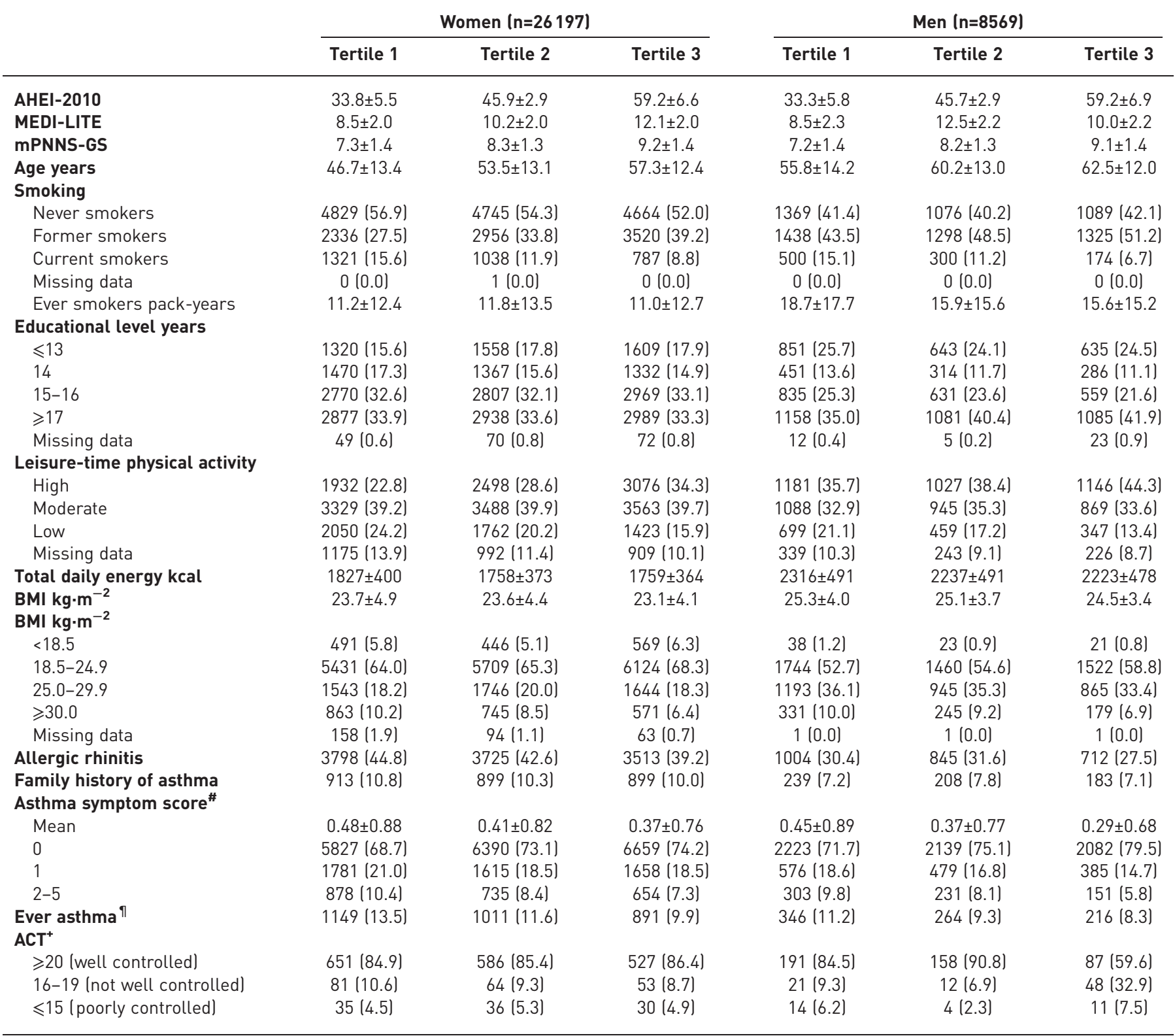

Data are presented as $\mathrm{n}(\%)$ or mean \pm SD unless otherwise stated. MEDI-LITE: literature-based adherence score to Mediterranean Diet; mPNNS-GS: modified Programme National Nutrition Santé Guideline Score; BMI: body mass index; ACT: asthma control test. \#: number of respiratory symptoms (1. breathless while wheezing; 2 . woken up with chest tightness; 3 . attack of shortness of breath at rest; 4 . attack of shortness of breath after exercise; and 5. woken by attack of shortness of breath) during the past 12 months. Each item is scored from zero to one and the total asthma symptom score ranges from zero to five. " : defined by at least one positive answer to the question "Have you ever had asthma?" in main questionnaires (baseline or follow-up) and by a positive answer to the question "Have you ever had an asthma attack?" or "Have you ever had an attack of shortness of breath at rest with wheezing?" in the respiratory survey (2016). ${ }^{+}$: test conducted among 2063 women and 546 men, based on five questions in the last 4 weeks on: 1. activity limitations (does asthma keep you from getting as much done at work, school or at home, "some of the time" to "all of the time"); 2. shortness of breath ("three to six times per week" to "more than once daily"); 3. woken up by asthma symptoms at night ("once per week" to "every night"); 4. use of a $\beta$-agonist inhaler ("two times per week" to "three or more times daily"); and 5. self-rated asthma control ("somewhat controlled" to "not controlled at all"). Each item is scored from one to five and the total ACT score ranges from five to 25 .

more likely ever smokers, had a lower educational level, practiced less moderate physical activity and were more often obese when compared to participants with controlled asthma (see supplementary table S7).

For men, after adjustments for potential confounders, we reported a negative and significant association between uncontrolled asthma and a greater adherence to the AHEI-2010 diet score. A borderline 
significant association was reported in women $(\mathrm{p}=0.06)$ (table 4). For the MEDI-LITE diet score we reported a negative significant association with uncontrolled asthma in women and a borderline significant association in men $(\mathrm{p}=0.10)$. When healthy diet was assessed by the mPNNS-GS score, negative associations were also reported between a greater adherence to the diet score and uncontrolled asthma, but significant this was only in men.

For AHEI-2010 and MEDI-LITE we reported an E-value of 2.08 in women. In men, E-values were 4.56 for AHEI-2010, 3.10 for MEDI-LITE and 5.91 for mPNNS-GS.

\section{Discussion}

In this large study of French adults (both women and men), we observed that higher dietary scores assessed by AHEI-2010, MEDI-LITE and mPNNS-GS were associated with a lower asthma symptom score. This association remained significant after controlling for several potential confounders and was also significant in participants without ever asthma. In addition, uncontrolled asthma was negatively associated with dietary behaviours corresponding to better adherence to the AHEI-2010 diet score, to the MEDI-LITE score among women in particular and to the mPNNS-GS score among men.

To our knowledge, very few studies have been published regarding the association between diet scores and asthma incidence $[9,12]$ or prevalence $[10]$ in adults. A study conducted in a large cohort of US women reported no association between diet quality (measured by AHEI-2010) and the risk of adult-onset asthma [9]; however, the authors defined asthma as a dichotomous trait which may not reflect its complexity [13]. By contrast, using the asthma symptom score in a case-control and family study on asthma with 20-years of follow-up, the authors reported a positive and significant association between a greater adherence to AHEI-2010 and improved asthma symptoms overtime in never smokers [12]. Our results are consistent with those based on the continuous definition of asthma, encouraging the use of the asthma score for future epidemiological studies on diet-asthma association. Besides longitudinal studies, a small case-control study in Australia (including 99 cases and 61 healthy controls) reported that participants with ever asthma had higher dietary inflammatory index scores, reflecting a more pro-inflammatory diet [10]. These results are also in agreement with ours, as greater AHEI-2010 and Mediterranean diet scores were associated with lower concentrations of inflammatory biomarkers [36].

Regarding secondary prevention, the identification of modifiable risk factors that could be targeted to help reduce asthma exacerbations and allow patients to reach a good level of asthma control, is of major importance. Asthma control is the main goal of asthma management and the ACT, an easy-to-use

TABLE 3 Associations between tertiles of dietary scores (the Alternate Healthy Eating Index 2010 (AHEI-2010), the literature-based adherence score to Mediterranean diet (MEDI-LITE) and the modified Programme National Nutrition Santé Guideline Score (mPNNS-GS)) and asthma symptom score (as a continuous variable) among women and men from the NutriNet-Santé study $(n=34766)$

\begin{tabular}{|c|c|c|c|c|c|c|c|c|}
\hline & \multicolumn{4}{|c|}{ Women ( $n=26$ 197) } & \multicolumn{4}{|c|}{ Men (n=8569) } \\
\hline & Tertile 1 & Tertile 2 & Tertile 3 & p-value ${ }^{\#}$ & Tertile 1 & Tertile 2 & Tertile 3 & p-value \\
\hline \multicolumn{9}{|l|}{ AHEI-2010 } \\
\hline $\mathrm{Mean}^{+}$ & $33.8 \pm 5.5$ & $45.9 \pm 2.9$ & $59.2 \pm 6.6$ & & $33.3 \pm 5.8$ & $45.7 \pm 2.9$ & $59.2 \pm 6.9$ & \\
\hline Age adjusted OR & 1.00 (ref) & $0.83(0.79-0.88)$ & $0.76(0.71-0.80)$ & $<0.0001$ & 1.00 (ref) & $0.81(0.73-0.90)$ & $0.63(0.56-0.70)$ & $<0.0001$ \\
\hline Further adjusted OR & 1.00 (ref) & $0.85(0.80-0.90)$ & $0.79(0.75-0.84)$ & $<0.0001$ & 1.00 (ref) & $0.82(0.75-0.91)$ & $0.67(0.60-0.75)$ & $<0.0001$ \\
\hline Mean $^{+}$ & $7.6 \pm 1.4$ & $10.5 \pm 0.5$ & $13.2 \pm 1.2$ & & $7.5 \pm 1.2$ & $10.5 \pm 0.5$ & $13.3 \pm 1.1$ & \\
\hline Age adjusted OR & 1.00 (ref) & $0.85(0.80-0.90)$ & $0.77(0.73-0.82)$ & $<0.0001$ & 1.00 (ref) & $0.80(0.71-0.89)$ & $0.68(0.61-0.76)$ & $<0.0001$ \\
\hline Further adjusted OR & 1.00 (ref) & $0.86(0.81,0.91)$ & $0.79(0.75,0.84)$ & $<0.0001$ & 1.00 (ref) & $0.81(0.73,0.91)$ & $0.72(0.65,0.80)$ & $<0.0001$ \\
\hline \multicolumn{9}{|l|}{ mPNNS-GS } \\
\hline Subjects & 8780 & 8269 & 9148 & & 3307 & 2674 & 2588 & \\
\hline $\mathrm{Mean}^{+}$ & $6.6 \pm 0.8$ & $8.3 \pm 0.4$ & $10.0 \pm 0.8$ & & $6.5 \pm 0.9$ & $8.3 \pm 0.4$ & $9.9 \pm 0.7$ & \\
\hline
\end{tabular}

Data are presented as $\mathrm{n}$, mean $\pm \mathrm{SD}$, or odds ratio with $95 \%$ confidence interval unless otherwise stated. ${ }^{*}: \mathrm{p}$-value for trend; ${ }^{\text {I: }}$ models were further adjusted for smoking, pack-years lamong ever smokers), educational level, leisure-time physical activity, total daily energy, allergic rhinitis and family history of asthma; ${ }^{+}$: total score within each tertile. 


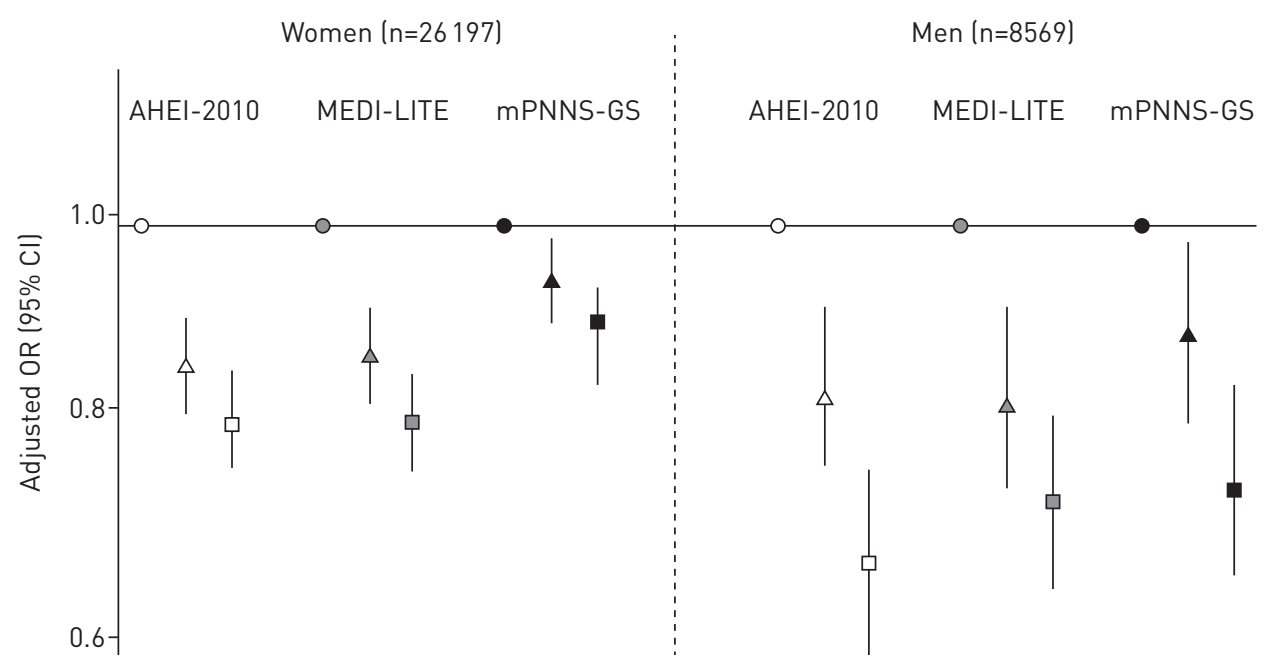

FIGURE 2 Associations between tertiles of dietary scores (the Alternate Healthy Eating Index 2010 (AHEI-2010), the literature-based adherence score to Mediterranean diet (MEDI-LITE) and the modified Programme National Nutrition Sante Guideline Score (mPNNS-GS)) and the asthma symptom score among women and men. Models were adjusted for age, smoking, pack-years, educational level, leisure-time physical activity, total daily energy, allergic rhinitis and family history of asthma. Circles are for tertile 1 of dietary scores (reference), triangles are for tertile 2 and squares are for tertile 3. Asthma symptom scores range from 0 to 5 . For trends, $p<0.001$ for all models.

instrument to evaluate the level of asthma control, has already been administered via the internet in other epidemiological studies $[37,38]$. Our results are consistent with those reported in other studies using different dietary scores $[7,11]$, suggesting that following a healthy diet probably contributes to better control of asthma. For instance, BARRos et al. [7] found that, in their study conducted in Portugal, a better quality diet corresponding to higher adherence to the alternate Mediterranean diet was associated with better asthma control. In addition, a pilot randomised controlled trial (RCT) in the US reported that 6 months of healthy eating intervention based on the Dietary Approaches to Stop Hypertension score, which emphasises fresh fruit, vegetables and low-fat dairy products, was associated with better asthma

TABLE 4 Associations between tertiles of dietary scores (the Alternate Healthy Eating Index 2010 (AHEI-2010), the literature-based adherence score to Mediterranean diet (MEDI-LITE) and the modified Programme National Nutrition Santé Guideline Score (mPNNS-GS)] and asthma control test (ACT) score among women and men from the NutriNet-Santé study (n=2609)

Women $(n=2063)$

\section{Tertile 1}

Tertile 3
Men (n=546)

\begin{tabular}{|c|c|c|c|c|c|c|c|c|}
\hline \multicolumn{9}{|l|}{ AHEI-2010 } \\
\hline Subjects & 767 & 686 & 610 & & 226 & 174 & 146 & \\
\hline $\mathrm{Mean}^{+}$ & $33.6 \pm 5.6$ & $45.8 \pm 2.8$ & $58.6 \pm 6.4$ & & $32.8 \pm 6.1$ & $45.8 \pm 2.8$ & $58.7 \pm 6.3$ & \\
\hline Age adjusted OR & 1.00 (ref) & $0.84(0.62-1.13)$ & $0.72(0.52-1.00)$ & 0.05 & 1.00 (ref) & $0.48(0.25-0.92)$ & $0.36(0.17-0.76)$ & 0.004 \\
\hline \multicolumn{9}{|l|}{ MEDI-LITE } \\
\hline Subjects & 815 & 648 & 600 & & 232 & 156 & 158 & \\
\hline Mean $^{+}$ & $7.5 \pm 1.4$ & $10.5 \pm 0.5$ & $13.2 \pm 1.2$ & & $7.3 \pm 1.5$ & $10.5 \pm 0.5$ & $13.3 \pm 1.3$ & \\
\hline \multicolumn{9}{|l|}{ mPNNS-GS } \\
\hline Subjects & 744 & 669 & 650 & & 230 & 193 & 123 & \\
\hline Mean $^{+}$ & $6.6 \pm 0.9$ & $8.3 \pm 0.4$ & $9.9 \pm 0.7$ & & $6.5 \pm 0.9$ & $8.3 \pm 0.4$ & $9.9 \pm 0.7$ & \\
\hline Age adjusted OR & 1.00 (ref) & $1.05(0.77-1.43)$ & $1.18(0.87-1.62)$ & 0.27 & 1.00 (ref) & $0.44(0.24-0.84)$ & $0.32(0.14-0.71)$ & 0.01 \\
\hline Further adjusted OR & 1.00 (ref) & $1.06(0.77-1.45)$ & $1.21(0.87-1.67)$ & 0.26 & 1.00 (ref) & $0.44(0.23-0.83)$ & $0.31(0.14-0.71)$ & 0.002 \\
\hline
\end{tabular}

Data are presented as $n$, mean $\pm S D$, or odds ratio with $95 \%$ confidence interval unless otherwise stated. ${ }^{\#}: p$-value for trend; ${ }^{\text {n: }}$ models were further adjusted for smoking, pack-years lamong ever smokers), educational level, leisure-time physical activity and total daily energy; ${ }^{+}$: total score within each tertile. 
control among adults with persistent asthma [11]. By contrast, a small RCT $(n=38)$ conducted in New Zealand [8] reported no improvement in asthma control over 12 weeks for the interventional groups encouraged to adopt a Mediterranean diet; however, this might be attributable to the small sample size and the short follow-up time.

Regarding potential mechanisms, it has been extensively reported that a better quality diet (high in fruits, vegetables and legumes) is associated with lower inflammatory biomarkers [39]. However, besides the well-known anti-oxidant and anti-inflammatory hypotheses in diet-asthma association [40], a recent hypothesis has been raised involving an imbalance in gut microbiota [41], in particular through a reduction in short-chain fatty acids (SCFAs) [41]. Higher levels of SCFAs, produced by bacteria in the gut during fermentation of insoluble fibre from dietary plant matter, were recently associated with a healthy diet as defined by a high intake of fruit, vegetables and legumes (i.e. high fibre content foods) [42] and were also reported to reduce airway inflammation [43]. In this manner, dietary intervention based on a fibre-rich diet might be relevant for both primary and secondary prevention of asthma.

The main strengths of our study include a large sample size, which allowed us to account for several potential confounders and to perform stratified analyses, the use of validated tools to assess asthma symptoms and asthma control, and the quality of the dietary data (based on at least three repeated $24 \mathrm{~h}$ dietary records over the last 8 years) reflecting the participants' food habits. Moreover, we used three dietary scores to evaluate overall diet quality, allowing us to take into account complex interactions between nutrients. Indeed, the three dietary scores are fairly correlated and it was important to observe consistent results since the three dietary scores are different measures of the same concept. The AHEI-2010 and Mediterranean diet scores, already studied in association with asthma and therefore allowing comparison with previous studies [7-9, 12], are both characterised by high intakes of fruit, vegetables and whole grains, moderate intake of alcohol and low intake of meat and processed meat. In addition, we assessed for the first time the mPNNS-GS score in relation to asthma, reflecting greater adherence to the French nutritional guidelines (which are consistent with international guidelines). Although the mPNNS-GS also reflects nutritional quality of diet, its construction differs slightly from those of the AHEI-2010 and MEDI-LITE scores. For instance, fruit and vegetable intakes were combined in the mPNNS-GS whereas they were investigated separately in the other two scores. Similarly, while "bread, cereals, potatoes and legumes" are combined in the mPNNS-GS (despite having different nutrient profiles, e.g. fibre content), the AHEI-2010 only combined "legumes and nuts", while the MEDI-LITE investigated "legumes" separately. Therefore, as healthier diet (as assessed by dietary scores) and higher overall diet quality in general is associated with fruit and vegetable intake, the difference in mPNNS-GS construction could have led to different results when assessing the association between diet and asthma (as observed for association between mPNNS-GS and asthma control among women).

We acknowledge a few potential limitations of our study. First, the cross-sectional nature of our respiratory data limits the interpretation of our results. Secondly, our data were collected from self-reported questionnaires, which are inherently prone to biases and should be interpreted with caution. However, self-reporting is widely used in epidemiological studies and, in the context of the NutriNet-Santé cohort, several validation studies have been performed to evaluate the accuracy of self-reported data as compared to "objective" measurement (for diet and anthropometric measurements) [24, 44, 45]. Furthermore, we used validated international tools to estimate asthma symptoms and asthma control. Thirdly, potential overlap between asthma and COPD might contribute to the association between asthma and diet scores, as the AHEI-2010 has already been found to be associated with COPD [9]. However, this is unlikely in the current study since similar associations were observed according to smoking. Fourthly, we acknowledge that participants from the NutriNet-Santé cohort, all volunteers involved in a long-term study investigating the association between nutrition and health, had more health-conscious behaviours and higher socio-professional and educational level overall [46]. As such, when compared to participants from a French representative sample of adults (Etude Nationale Nutrition Santé), participants from the NutriNet-Santé cohort reported a higher intake of healthy foods [46]. As unhealthy dietary behaviours are underrepresented in our study, the strength of the diet-asthma association is likely weakened in our convenient sample as compared to the general population. Furthermore, like previous studies on the dietasthma association based on convenient samples [47, 48], our study is looking for aetiology rather than for description of population health and therefore representativeness is less important than contrast between groups of exposure. Lastly, we acknowledge that the observed association could partly be explained by unmeasured or residual confounding. However, analyses were adjusted for several potential confounders and we further calculated the E-values as recently proposed [34]. Using the E-values, we found that an unmeasured confounder needs to be associated with both diet and asthma by a risk ratio of roughly 2.0 (at least) to explain away the association, which we believe is unlikely. 
In summary, our results support the hypothesis that healthy diet, an important contributor to good health, might also be relevant in prevention of asthma and its lack of control. Our results extend and justify the need to continually propagate public health recommendations on promoting healthy diet behaviour.

Acknowledgements: The authors thank Nathalie Druesne-Pecollo and Thi Duong Van (Equipe de Recherche en Epidémiologie Nutritionnelle (EREN), Centre d'Epidémiologie et Statistiques Sorbonne Paris Cité, Inserm (U1153), Inra (U1125), Bobigny, France) for their contribution to the development and implementation of the respiratory survey in the Nutrinet-Santé study. We also thank Younes Esseddik, Frédéric Coffinieres, Régis Gatibelza and Paul Flanzy (computer scientists) and Nathalie Arnault, Véronique Gourlet, Fabien Szabo, Julien Allegre and Laurent Bourhis (data managers/biostatisticians) (Equipe de Recherche en Epidémiologie Nutritionnelle (EREN), Centre d'Epidémiologie et Statistiques Sorbonne Paris Cité, Inserm (U1153), Inra (U1125), Bobigny, France) for their technical contribution to the NutriNet-Santé study. We thank all the volunteers of the NutriNet-Santé cohort.

Author contributions: R.M. Andrianasolo, S. Hercberg, P. Galan, E. Kesse-Guyot and R. Varraso designed and conducted the research; E. Kesse-Guyot, M. Adjibade, S. Hercberg, P. Galan and R. Varraso provided essential reagents or provided essential materials; R.M. Andrianasolo and R. Varraso analysed data or performed statistical analysis; R.M. Andrianasolo, P. Galan and R. Varraso wrote the manuscript and had primary responsibility for final content; R.M. Andrianasolo, E. Kesse-Guyot, M. Adjibade, S. Hercberg, P. Galan, and R. Varraso were involved in interpreting the results and editing the manuscript for important intellectual content; all authors read, edited and approved the final manuscript.

Conflict of interest: None declared.

Support statement: The NutriNet-Santé Study was supported by the following public institutions: Ministère de la Santé, Santé Publique France, Institut National de la Santé et de la Recherche Médicale, Institut National de la Recherche Agronomique, Conservatoire National des Arts et Métiers and Université Paris 13.

\section{References}

1 Vos T, Allen C, Arora M, et al. Global, regional, and national incidence, prevalence, and years lived with disability for 310 diseases and injuries, 1990-2015: a systematic analysis for the Global Burden of Disease Study 2015. The Lancet 2016; 388: 1545-1602.

2 Devereux G. The increase in the prevalence of asthma and allergy: food for thought. Nat Rev Immunol 2006; 6 : 869-874.

3 Garcia-Larsen V, Del Giacco SR, Moreira A, et al. Asthma and dietary intake: an overview of systematic reviews. Allergy 2016; 71: 433-442.

$4 \quad$ Varraso R. Nutrition and asthma. Curr Allergy Asthma Rep 2012; 12: 201-210.

5 Berthon B, Wood L. Nutrition and respiratory health-feature review. Nutrients 2015; 7: 1618-1643.

6 Hu FB. Dietary pattern analysis: a new direction in nutritional epidemiology. Curr Opin Lipidol 2002; 13: 3-9.

7 Barros R, Moreira A, Fonseca J, et al. Adherence to the Mediterranean diet and fresh fruit intake are associated with improved asthma control. Allergy 2008; 63: 917-923.

8 Sexton P, Black P, Metcalf P, et al. Influence of Mediterranean diet on asthma symptoms, lung function, and systemic inflammation: a randomized controlled trial. J Asthma 2013; 50: 75-81.

9 Varraso R, Chiuve SE, Fung TT, et al. Alternate Healthy Eating Index 2010 and risk of chronic obstructive pulmonary disease among US women and men: prospective study. BMJ 2015; 350: h286.

10 Wood LG, Shivappa N, Berthon BS, et al. Dietary inflammatory index is related to asthma risk, lung function and systemic inflammation in asthma. Clin Exp Allergy 2015; 45: 177-183.

11 Ma J, Strub P, Lv N, et al. Pilot randomised trial of a healthy eating behavioural intervention in uncontrolled asthma. Eur Respir J 2016; 47: 122-132.

12 Li Z, Kesse-Guyot E, Dumas $\mathrm{O}$, et al. Longitudinal study of diet quality and change in asthma symptoms in adults, according to smoking status. Br J Nutr 2017; 117: 562-571.

13 Pekkanen J. Operational definitions of asthma in studies on its aetiology. Eur Respir J 2005; 26: 28-35.

14 Sunyer J, Pekkanen J, Garcia-Esteban R, et al. Asthma score: predictive ability and risk factors. Allergy 2007; 62: 142-148.

15 Global Initiative for Asthma. Global Strategy for Asthma Management and Prevention (2017 Update). http:// ginasthma.org/wp-content/uploads/2016/01/wms-GINA-2017-main-report-tracked-changes-for-archive.pdf

16 Sofi F, Dinu M, Pagliai G, et al. Validation of a literature-based adherence score to Mediterranean diet: the MEDI-LITE score. Int J Food Sci Nutr 2017; 68: 757-762.

17 Reedy J, Krebs-Smith SM, Miller PE, et al. Higher diet quality is associated with decreased risk of all-cause, cardiovascular disease, and cancer mortality among older adults. J Nutr 2014; 144: 881-889.

18 Harmon BE, Boushey CJ, Shvetsov YB, et al. Associations of key diet-quality indexes with mortality in the Multiethnic Cohort: the Dietary Patterns Methods Project. Am J Clin Nutr 2015; 101: 587-597.

19 Sofi F, Abbate R, Gensini GF, et al. Accruing evidence on benefits of adherence to the Mediterranean diet on health: an updated systematic review and meta-analysis. Am J Clin Nutr 2010; 92: 1189-1196.

20 Estaquio C, Kesse-Guyot E, Deschamps V, et al. Adherence to the French Programme National Nutrition Santé Guideline Score is associated with better nutrient intake and nutritional status. J Am Diet Assoc 2009; 109: 1031-1041.

21 Hercberg S, Chat-Yung S, Chaulia M. The French National Nutrition and Health Program: 2001-2006-2010. Int J Public Health 2008; 53: 68-77.

22 Estaquio C, Castetbon K, Kesse-Guyot E, et al. The French National Nutrition and Health Program score is associated with nutritional status and risk of major chronic diseases. J Nutr 2008; 138: 946-953.

23 Hercberg S, Castetbon K, Czernichow S, et al. The Nutrinet-Santé Study: a web-based prospective study on the relationship between nutrition and health and determinants of dietary patterns and nutritional status. BMC Public Health 2010; 10: 242. 
24 Lassale $\mathrm{C}$, Castetbon $\mathrm{K}$, Laporte $\mathrm{F}$, et al. Validation of a web-based, self-administered, non-consecutive-day dietary record tool against urinary biomarkers. Br J Nutr 2015; 113: 953-962.

25 Etude Nutrinet-Santé. Table de composition des aliments de l'étude Nutrinet-Santé (Nutrinet-Santé Study Food Composition Database). Paris, Economica, 2013.

26 Chiuve SE, Fung TT, Rimm EB, et al. Alternative dietary indices both strongly predict risk of chronic disease. J Nutr 2012; 142: 1009-1018.

27 Burney PG, Luczynska C, Chinn S, et al. The European Community respiratory health survey. Eur Respir J 1994; 7: 954-960.

28 Schatz M, Sorkness CA, Li JT, et al. Asthma control test: reliability, validity, and responsiveness in patients not previously followed by asthma specialists. J Allergy Clin Immunol 2006; 117: 549-556.

29 Vergnaud A-C, Touvier M, Méjean C, et al. Agreement between web-based and paper versions of a socio-demographic questionnaire in the NutriNet-Santé Study. Int J Public Health 2011; 56: 407-417.

30 WHO Technical Report Series (No. 894). Obesity: preventing and managing the global epidemic. Report of a WHO Consultation. Geneva, World Health Organization, 2000. http://apps.who.int/iris/handle/10665/42330

31 Hagströmer M, Oja P, Sjöström M. The International Physical Activity Questionnaire (IPAQ): a study of concurrent and construct validity. Public Health Nutr 2006; 9: 755-762.

32 Fuseini H, Newcomb DC. Mechanisms driving gender differences in asthma. Curr Allergy Asthma Rep 2017; 17: 19.

33 Jacquemin B, Sunyer J, Forsberg B, et al. Association between modelled traffic-related air pollution and asthma score in the ECRHS. Eur Respir J 2009; 34: 834-842.

34 VanderWeele TJ, Ding P. Sensitivity analysis in observational research: introducing the E-Value. Ann Intern Med 2017; 167: 268-274.

35 Yuan YC. Multiple imputation for missing data: concepts and new development (paper 267-25). In: Proceedings of the Twenty-Fifth Annual SAS Users Group International Conference. Cary, SAS Institute, 2000.

36 Fung TT, McCullough ML, Newby PK, et al. Diet-quality scores and plasma concentrations of markers of inflammation and endothelial dysfunction. Am J Clin Nutr 2005; 82: 163-173.

37 Peters SP, Jones CA, Haselkorn T, et al. Real-world evaluation of asthma control and treatment (REACT): findings from a national web-based survey. J Allergy Clin Immunol 2007; 119: 1454-1461.

38 Dumas O, Wiley A, Quinot C, et al. Occupational exposure to disinfectants and asthma control in U.S. nurses. Eur Respir J. 2017; 50: 1700237.

39 Ahluwalia N, Andreeva VA, Kesse-Guyot E, et al. Dietary patterns, inflammation and the metabolic syndrome. Diabetes Metab 2013; 39: 99-110.

40 Caramori G, Papi A. Oxidants and asthma. Thorax 2004; 59: 170-173.

41 McKenzie C, Tan J, Macia L, et al. The nutrition-gut microbiome-physiology axis and allergic diseases. Immunol Rev 2017; 278: 277-295.

42 De Filippis F, Pellegrini N, Vannini L, et al. High-level adherence to a Mediterranean diet beneficially impacts the gut microbiota and associated metabolome. Gut 2016; 65: 1812-1821.

43 Halnes I, Baines K, Berthon B, et al. Soluble fibre meal challenge reduces airway inflammation and expression of GPR43 and GPR41 in asthma. Nutrients 2017; 9: 57.

44 Lassale $\mathrm{C}$, Péneau S, Touvier $\mathrm{M}$, et al. Validity of web-based self-reported weight and height: results of the Nutrinet-Santé study. J Med Internet Res 2013; 15: e152.

45 Lassale C, Castetbon K, Laporte F, et al. Correlations between fruit, vegetables, fish, vitamins, and fatty acids estimated by web-based nonconsecutive dietary records and respective biomarkers of nutritional status. $J$ Acad Nutr Diet 2016; 116: 427-438.e5.

46 Andreeva VA, Deschamps V, Salanave B, et al. Comparison of dietary intakes between a large online cohort study (Etude NutriNet-Santé) and a nationally representative cross-sectional study (Etude Nationale Nutrition Santé) in France: addressing the issue of generalizability in e-epidemiology. Am J Epidemiol 2016; 184: 660-669.

47 Bao Y, Bertoia ML, Lenart EB, et al. Origin, methods, and evolution of the Three Nurses' health studies. Am J Public Health 2016; 106: 1573-1581.

48 Clavel-Chapelon F, E3N Study Group. Cohort profile: the French E3N cohort study. Int J Epidemiol. 2015; 44: 801-809. 